\title{
Aeromonas schubertii
}

National Cancer Institute

\section{Source}

National Cancer Institute. Aeromonas schubertii. NCI Thesaurus. Code C86129.

A species of Gram negative, rod shaped bacteria assigned to the phylum Proteobacteria. This bacteria is oxidase positive, hemolytic, does not hydrolyze esculin, produces arginine dihydrolase and is negative for indole production. A. schubertii is found in aquatic environments and is pathogenic. 